Received: 6 March 2017

Accepted: 8 June 2017

Published online: 28 June 2017

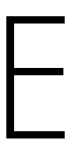

C NTF
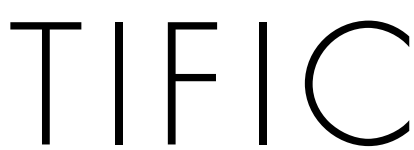

REP

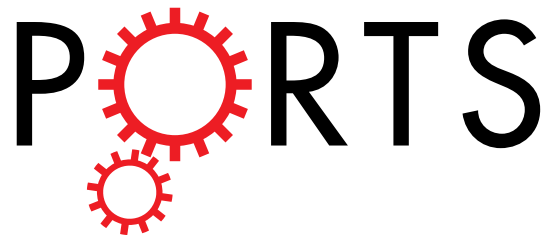

\title{
OPEN
}

\section{Ultra-broadband enhancement of nonlinear optical processes from randomly patterned super absorbing metasurfaces}

Nan Zhang ${ }^{1}$, Ziheng $\mathrm{Ji}^{2}$, Alec R. Cheney ${ }^{1}$, Haomin Song ${ }^{1}$, Dengxin $\mathrm{Ji}^{1}$, Xie Zeng ${ }^{1}$, Borui Chen ${ }^{1}$, Tianmu Zhang ${ }^{1}$, Alexander N. Cartwright ${ }^{1}$, Kebin Shi ${ }^{2}$ \& Qiaoqiang Gan ${ }^{1}$

Broadband light trapping and field localization is highly desired in enhanced light-matter interaction, especially in harmonic generations. However, due to the limited resonant bandwidth, most periodic plasmonic nanostructures cannot cover both fundamental excitation wavelength and harmonic generation wavelength simultaneously. Therefore, most previously reported plasmonic nonlinear optical processes are low in conversion efficiency. Here, we report a strong enhancement of second harmonic generation based on a three-layered super absorbing metasurface structure consisting of a dielectric spacer layer sandwiched by an array of random metallic nanoantennas and a metal ground plate. Intriguingly, the strong light trapping band (e.g. $>80 \%$ ) was realized throughout the entire visible to near-infrared spectral regime (i.e., from $435 \mathrm{~nm}$ to $1100 \mathrm{~nm}$ ), enabling plasmonically enhanced surface harmonic generation and frequency mixing across a broad range of excitation wavelengths, which cannot be achieved with narrow band periodic plasmonic structures. By introducing hybrid random antenna arrays with small metallic nanoparticles and ultra-thin nonlinear optical films (e.g. $\mathrm{TiO}_{2}$ ) into the nanogaps, the nonlinear optical process can be further enhanced. This broadband lighttrapping metastructure shows its potential as a building block for emerging nonlinear optical metaatoms.

Nonlinear light-matter interaction at mesoscopic scales has emerged as an intriguing platform for studying fundamental optical physics and developing practical photonic applications $s^{1,2}$. However, the general light-matter interaction efficiency of many conventional nonlinear optical processes is low ${ }^{3,4}$. Therefore, high intensity lasers are usually required in conventional nonlinear optical experiments. As a result, plasmonics, which leverages the resonant interaction between light and free electrons of metallic nanostructures, has garnered interest as a means to confine electromagnetic fields within nanoscale volumes and significantly enhance localized field ${ }^{5,6}$. For instance, periodic patterns were utilized in three-layered metamaterial super absorbers that were reported to enhance the second harmonic generation (SHG) signal with unique electronic tunability ${ }^{7}$. However, periodic nanostructures and other specifically designed nanopatterns rely on top-down lithographic techniques (e.g. focused ion beam milling ${ }^{8,9}$, electronic-beam lithography ${ }^{10,11}$, nanoimprint lithography ${ }^{12}$ and combined hybrid fabrication methods ${ }^{13,14}$ ), imposing serious cost barriers for practical applications. In addition, according to the definition of the enhancement factor (i.e., $\left.E F=\left(\frac{E_{\text {local }}(\omega)}{E_{0}(\omega)}\right)^{2}\left(\frac{E_{\text {local }}(2 \omega)}{E_{0}(2 \omega)}\right)^{2}{ }^{15}\right)$, an ideal structure to enhance the SHG is to enhance the localized field at excitation and emission wavelengths simultaneously within the same surface structure. Unfortunately, due to the narrow resonant nature of periodic structures, the enhanced spectral range is usually limited, that is insufficient to cover both excitation and emission wavelengths. Thus, broadband light trapping and localization are essential in the development of practical on-chip optoelectronic circuits that leverage nonlinear optical processes in their operation.

${ }^{1}$ Department of Electrical Engineering, The State University of New York at Buffalo, Buffalo, NY, 14260, USA. ${ }^{2}$ State Key Laboratory for Mesoscopic Physics, Collaborative Innovation Center of Quantum Matter, School of Physics, Peking University, Beijing, 100871, China. Nan Zhang, Ziheng Ji and Alec R. Cheney contributed equally to this work. Correspondence and requests for materials should be addressed to K.S. (email: kebinshi@pku.edu.cn) or Q.G. (email: qqgan@buffalo.edu) 
(a)

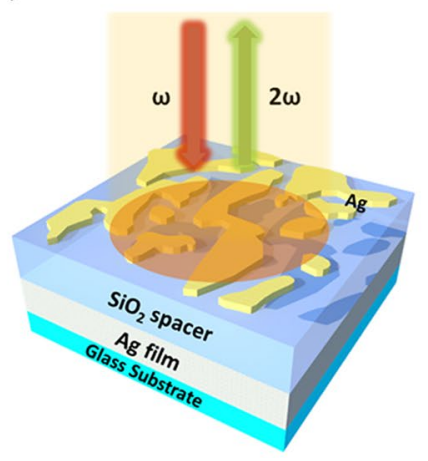

(b)

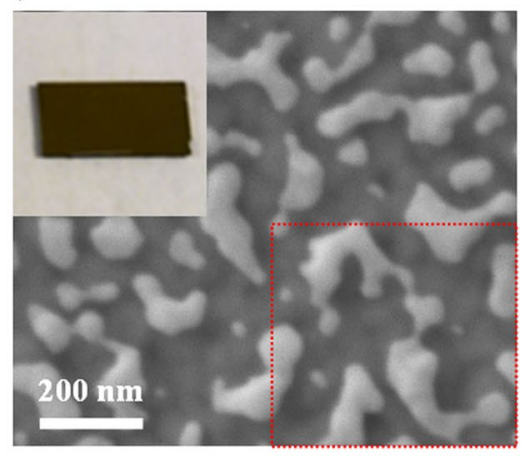

(c)

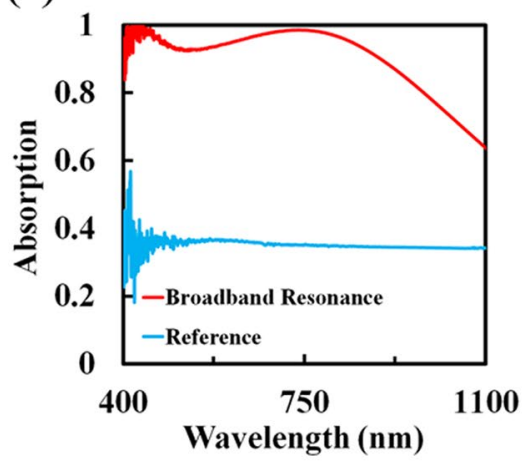

Figure 1. (a) Schematic of the designed three-layered absorbing metasurface. (b) SEM image of top random Ag NPs. Inset: Photograph of the ultra-broadband super absorbing metasurface. (c) Absorption spectra of the three-layered absorber (red curve) and the reference structure (blue curve).

To address this challenge, a simple, low-cost, scalable, lithography-free method to manufacture super-absorbing metasurfaces was recently developed ${ }^{16}$. By controlling the average geometric parameters of directly deposited random metal nanoparticles (NPs) with a low degree of symmetry, a super-absorbing plasmonic metamaterial structure was realized with broadband resonant spectral tunability (e.g. $>80 \%$ absorption band from $414 \mathrm{~nm}$ to $956 \mathrm{~nm}$ ). In this case, when the strongly trapped light interacts with these random nanoantennas, it resonantly couples to free-electrons at the edges of NPs with the appropriate geometry. We have recently shown that a single piece of such a "metasurface" can be used as a universal substrate for excitation wavelengths $\left(\lambda_{\mathrm{ex}}\right)$ lying within its light trapping band for applications that leverage surface enhanced light-matter interaction, specifically surface-enhanced Raman spectroscopy (SERS) ${ }^{17}$. According to our previously reported experiment to resolve chemical molecules, an enhancement factor over $10^{7}$ was obtained. It should be noted that both SERS and plasmonically enhanced nonlinear optical effects share similar requirements in terms of strong local field enhancement at both excitation and emission wavelengths ${ }^{5}$. In particular, nonlinear optics require an even broader light trapping band: e.g. dual resonant SHG requires the localized field enhancement from $\lambda_{\text {ex }} / 2$ to $\lambda_{\mathrm{ex}}$, which is significantly broader than the required spectrum for SERS near a given $\lambda_{\mathrm{ex}}$. Therefore, broadband light-trapping metastructures will also behave as a novel platform for plasmonically enhanced surface harmonic generation and frequency mixing across a broad range of excitation wavelengths, which has not yet been achieved with narrow band periodic plasmonic structures. In this work, we report ultra-broadband SHG using metasurfaces engineered with random NP arrays, which show promise as a building block for emerging nonlinear optical meta-atoms $s^{6,7,18}$.

\section{Results and Discussion}

Ultra-broadband super absorbing metasurfaces. As illustrated in Fig. 1(a), our three-layered random metal-dielectric-metal (rMDM) super absorbing metasurface consists of a 200-nm-thick silver (Ag) film as a reflector, a 70-nm-thick silicon dioxide $\left(\mathrm{SiO}_{2}\right)$ spacer layer and a layer of Ag random NPs. Following our previously reported lithography-free fabrication technique ${ }^{16}$, direct Ag deposition followed by thermal annealing was used to manipulate the average morphology (size, spacing, and "interconnectedness") of the Ag NPs to tune the effective optical constant and realize the desired light-trapping band (see Methods for details of fabrication). In this experiment, the Ag NPs were formed by annealing a 14-nm-thick Ag film under $200^{\circ} \mathrm{C}$ for 1 hour. The morphology of this sample was characterized by scanning electronic microscope (SEM) as shown in Fig. 1(b), indicating that randomly distributed Ag NPs were directly obtained during the thermal annealing process. The inset of Fig. 1(b) shows a color photograph of the sample, where one can note the almost black appearance of the highly broadband absorbing metasurface. The optical absorption of the metasurface was characterized using a Fourier transform infrared spectrometer (FTIR, Bruker VERTEX 70) with an extended light source covering visible wavelengths. As shown by the red curve in Fig. 1(c), a strong resonant absorption peak of $98.6 \%$ was obtained at the wavelength of $736 \mathrm{~nm}$ with the $80 \%$ absorption band spanning from $400 \mathrm{~nm}$ to $980 \mathrm{~nm}$, which is significantly broader than the previous report ${ }^{19}$. For reference, we used a single Ag NP layer on glass substrate with no $\mathrm{SiO}_{2}$ spacer or Ag film. The reference optical absorption spectrum is shown by the blue curve in Fig. 1(c). One can see that the absorption of the metasurface at $736 \mathrm{~nm}$ is over $98 \%$ while the reference sample is only $35 \%$, indicating the enhanced light trapping in the metasurface structure. When the incident light is trapped in the metasurface, it is re-distributed between the random NPs, which results in significantly enhanced local fields for surface enhanced nonlinear optics. It should be noted that the morphologies of rMDM metasurface and the reference sample are similar due to the similar wettabilities at the interface of $\mathrm{Ag} / \mathrm{SiO}$-dielectric-layer and $\mathrm{Ag} /$ glass-substrate (see Section 1 in the supporting material for statistical analysis of the NP size distribution).

Nonlinear optical response of broadband metasurfaces. To demonstrate the enhancement of SHG in the metasurface, we studied the nonlinear optical properties of the random metasurface with the setup shown schematically in Fig. 2(a). The sample was irradiated with an ultrafast laser tunable from $740 \mathrm{~nm}$ to $1020 \mathrm{~nm}$, with the pulse length of $80 \mathrm{fs}$ and a repetition rate of $80 \mathrm{MHz}$, generated by using a broadband Ti-sapphire oscillator 
(a)

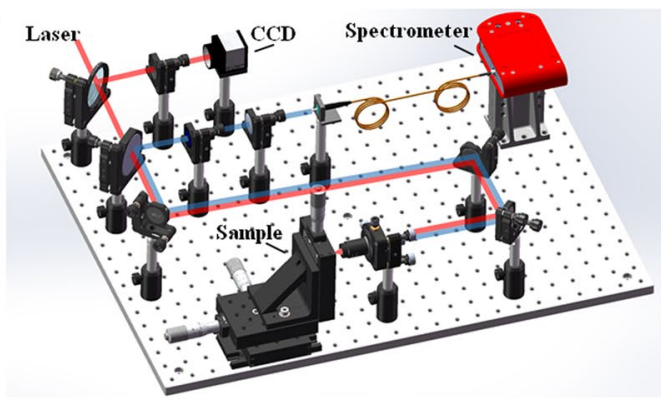

(c)

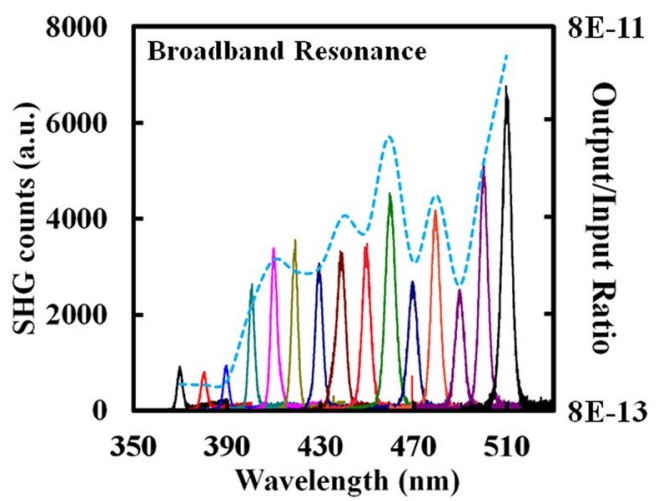

(b)

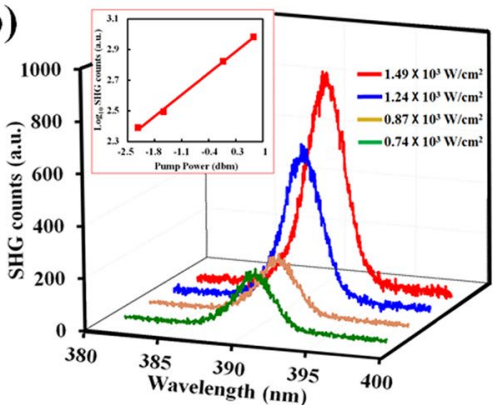

(d)

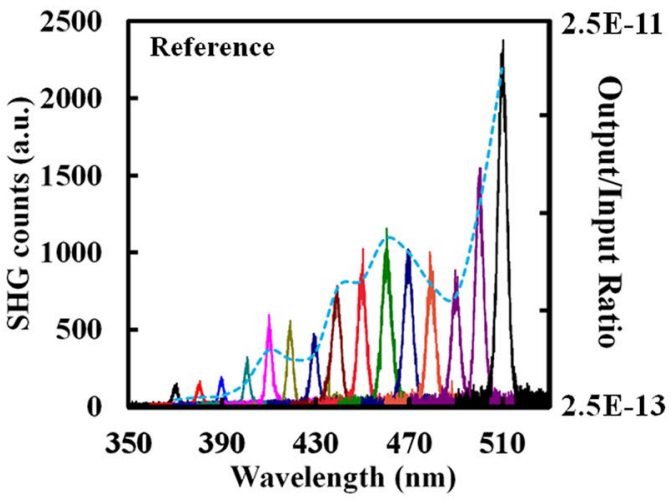

Figure 2. (a) Schematic of experimental setup for SHG measurement of fabricated metasurfaces. (b) Measured SHG intensities at different incident power. The inset is a log-log plot of amplitude of the SHG signal as a function of incident power. (c,d) Measured SHG intensities and SHG output/input ratios (blue dashed curve) versus incident wavelengths obtained with $(\mathbf{c})$ the broadband metasurface and (d) the reference sample, respectively.

(Spectra-Physics MaiTai) and an optical parametric oscillator (Spectra-Physics Inspire 100) (see Methods for details of SHG experimental setup). The average power of the fundamental beam was controlled by a continuously variable neutral density filter and set to $1.2 \mathrm{~mW}$ as measured at the sample position to avoid damage to the top NPs. The second harmonic signal was then collected in reflection mode and analyzed by a spectrometer (Acton SpectraPro, SP-2500) equipped with a liquid nitrogen cooled charge-coupled device (CCD). The diameter of the laser spot is approximately $8 \mu \mathrm{m}$. Figure $2(\mathrm{~b})$ shows the emission spectra generated using a focused $780 \mathrm{~nm}$ fundamental excitation beam with different average powers ranging from $0.74 \times 10^{3} \mathrm{~W} / \mathrm{cm}^{2}$ to $1.49 \times 10^{3} \mathrm{~W} / \mathrm{cm}^{2}$. An obvious signal at $390 \mathrm{~nm}$ was observed. The inset of Fig. 2(b) is a log-log plot of signal amplitude as a function of incident power, which exhibits a clear linear dependence with a slope of $\sim 2.02$. This corresponds to a quadratic dependence of signal on incident power, thus confirming the measured signals are from SHG (i.e., proportional to $\left.|E|^{2}\right)$.

Importantly, the broadband light-trapping of the metasurface with random nanoantennas should likewise result in a unique broadband SHG. To validate this broadband nonlinear optical response, we then leveraged the tunability of the ultra-fast laser to excite the same chip with different fundamental wavelengths. As shown in Fig. 2(c), when the excitation wavelength was tuned from $740 \mathrm{~nm}$ to $1020 \mathrm{~nm}$, obvious SHG signals from $370 \mathrm{~nm}$ to $510 \mathrm{~nm}$ were observed from the broadband metasurface (see Section 2 in the supporting material for details of SHG data calibration). In contrast, the reference sample (Fig. 2(d)) exhibited much smaller SHG intensities with the same input power, indicating an enhancement of light-matter interaction in the rMDM broadband super-absorbing metasurface.

Plasmonic mode matching in the random super absorbing metasurface. In most periodically patterned metallic structures for plasmonic nonlinear optics, spatial overlap of plasmon modes was typically not emphasized since highly periodic structures produce narrow resonance bands (e.g. refs 20-25). According to a recent report, sophisticated nanostructures were employed to realize two resonances at $\lambda_{\text {ex }} / 2$ and $\lambda_{\text {ex }}$ simultaneously with highly overlapped spatial mode distributions to improve the SHG efficiency ${ }^{26}$. In our broadband rMDM structure, the light trapping at $\lambda_{e x} / 2$ and $\lambda_{e x}$ is addressed satisfactorily. The remaining question is the spatial mode overlap. In random structures, depolarization introduced by hyper-Reyleigh scattering from random NPs will result in incoherency of SHG signals ${ }^{27-31}$. It is therefore difficult to manipulate the spatial mode distribution. Recently, a cavity was introduced to enhance the electric field at both fundamental and SHG wavelengths, and realized a spectral and spatial coincidence of $\mathrm{SH}$ response with the free excitons of $\mathrm{ZnO}$ nanorods ${ }^{32}$. It also indicates that an overlapped spatial mode is highly desired to improve the SHG efficiency. To interpret the mode overlap in our rMDM structure, we loaded a part of the SEM image in Fig. 1(b) (i.e., the area within the 
(a)

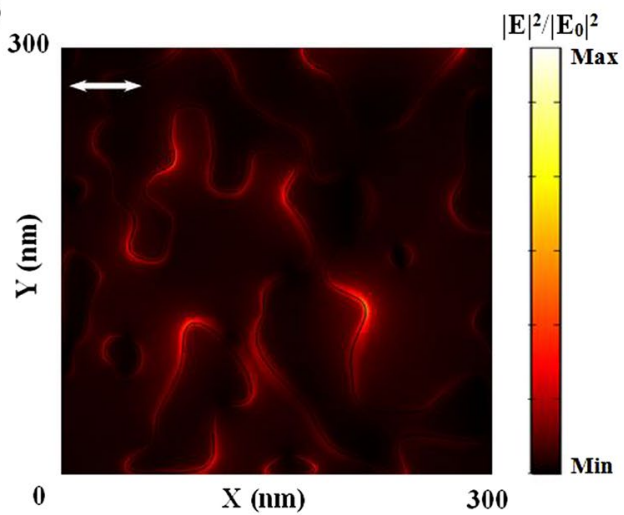

(b)

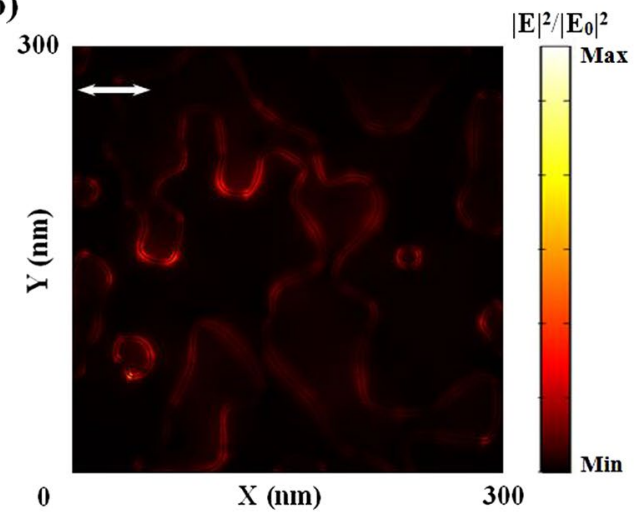

(d)

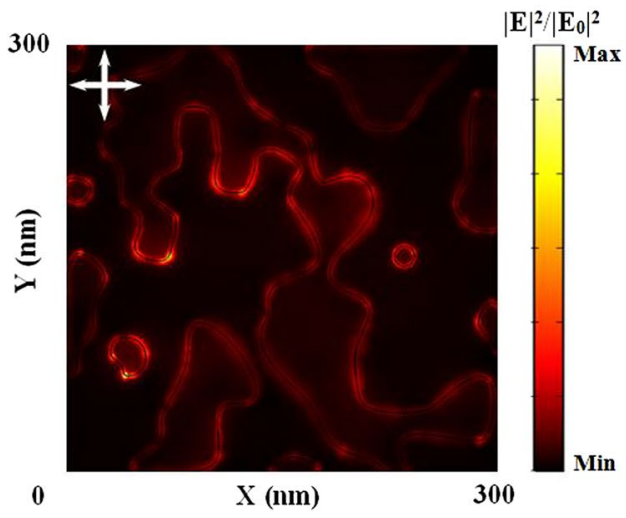

(c)

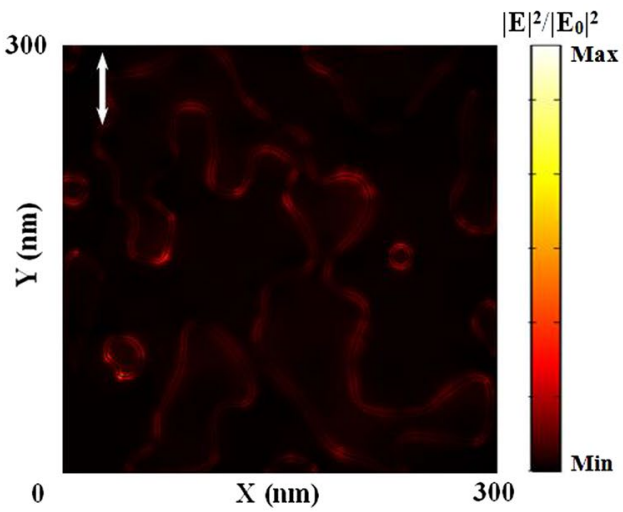

Figure 3. Modeled electric field enhancement distribution among NPs in the red dotted squares shown in Fig. 1(b) at $1000 \mathrm{~nm}$ wavelength with $\mathrm{x}$-polarization $(\mathbf{a})$, at $500 \mathrm{~nm}$ wavelength with $\mathrm{x}$-polarization $(\mathbf{b})$, at $500 \mathrm{~nm}$ wavelength with y-polarization (c), and at $500 \mathrm{~nm}$ wavelength with $\mathrm{x}$ - and y-polarization (d).

red dotted square) into the commercial software COMSOL, and modeled the spatial distribution of the electric field. The localized field distribution at the wavelength of $1000 \mathrm{~nm}$ under a linearly polarized excitation is shown in Fig. 3(a). In our experiment, we employed a polarizer to characterize the polarization dependence and confirmed that the SHG signal is non-polarized (see Section 3 in the supporting material for raw data). Therefore, the localized field distribution at $500 \mathrm{~nm}$ is the summation of $\mathrm{x}$ - (Fig. 3(b)) and y-polarized situation (Fig. 3 (c)), as shown in Fig. 3(d). To evaluate the mode matching situation, we calculated the correlation between Fig. 3(a and $b-d$ ). The correlation between these two mode distributions is $24.0 \%$ (calculated by comparing Fig. 3(a) with Fig. 3(b)), 21.4\% (calculated by comparing Fig. 3(a) with Fig. 3(c)), and 37.9\% (calculated by comparing Fig. 3(a) with Fig. 3(d)), respectively. Therefore, the depolarization effect for the SHG signal is actually beneficial with respect to mode matching requirements.

Further enhancement of SHG. It is generally believed that smaller gaps between metallic nanopatterns will result in stronger localized field due to optically driven free electrons coupled across the gap. In recent years, significant effort has been invested to reveal the upper limit for plasmonic enhancement using ultra-small gaps (e.g. refs 33-36), even approaching the quantum limit within subnanometer regions ${ }^{37-42}$. A straightforward subsequent question is how we can reduce the gap between our random NPs to further enhance the effective nonlinearity of the metasurface and their corresponding SHG signals. In using thermal annealing to control the morphology and size of surface NPs, it was observed that the spacing between adjacent NPs is also controllable. During the annealing process, metal atoms in the film migrate and aggregate to form islands ${ }^{43}$, and the void left in their absence defines the interparticle spacing. The more atoms aggregate, the larger interparticle spacing will form. Thus it can be seen that the spacing between smaller NPs is generally smaller than the spacing between larger NPs. With this in mind, we will discuss another multi-step direct deposition method to realize smaller gaps, as illustrated in Fig. 4(a). Starting with samples fabricated as described above, we then deposited a second Ag film, $5 \mathrm{~nm}$ thick to be below the percolation threshold of $\sim 6 \mathrm{~nm}^{44}$, on top of the random nanopattern that results from the aforementioned annealing process. Optical characterization, as shown by the purple curve in Fig. 4(b), revealed a red shift in the absorption peak from $736 \mathrm{~nm}$ to $908 \mathrm{~nm}$ due to multi-mode resonances ${ }^{45}$. A strong broadband absorption was maintained, with $>80 \%$ absorption bands both from $<400 \mathrm{~nm}$ to $472 \mathrm{~nm}$ and from $686 \mathrm{~nm}$ to $1100 \mathrm{~nm}$. Comparing SEM images before and after this second deposition (Fig. 4(c) and (d), respectively) confirmed that smaller NPs were formed in the area between large metal islands as expected. The average gap distances between NPs in this case are reduced to below $10 \mathrm{~nm}$, and therefore should support stronger localized field. To validate this prediction, we loaded a part of the SEM image of the top films (see the 
(a)

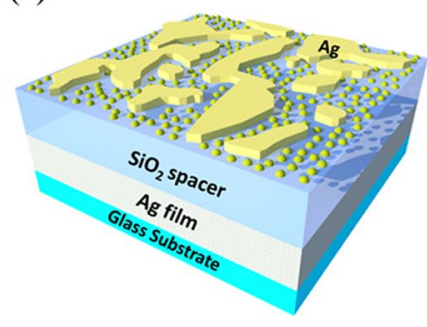

(e) (b)

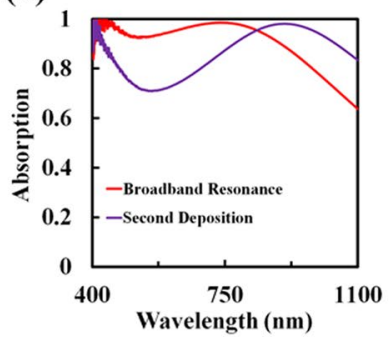

(c)

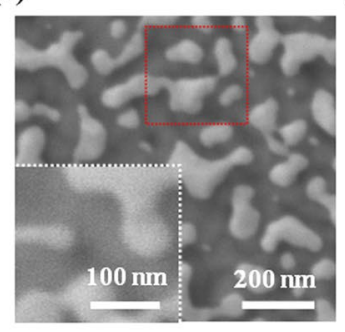

(d)

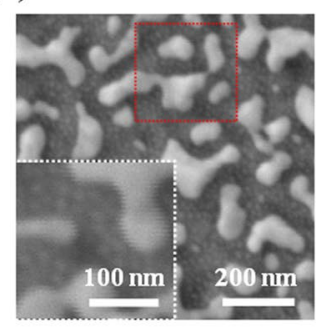

$|\mathbf{E}|^{2} /\left|\mathbf{E}_{0}\right|^{2} \quad$ (f)
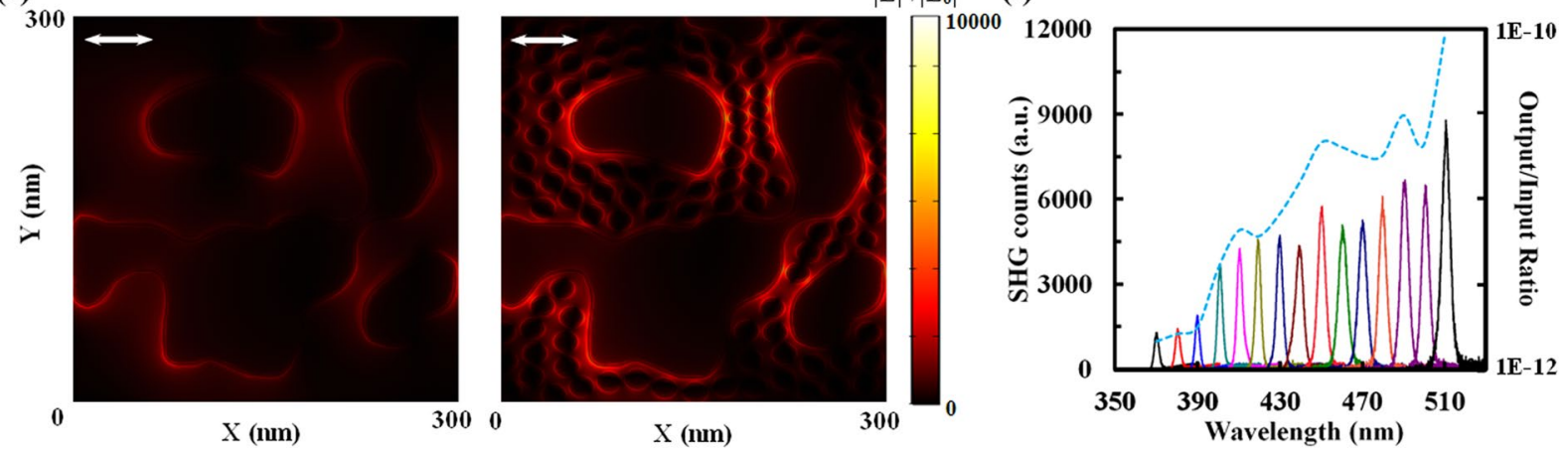

Figure 4. (a) Schematic of the designed three-layered absorbing metasurface after a multi-step deposition process. (b) Absorption spectra of the three-layered absorber before (red curve) and after the second-step deposition (purple curve). (c,d) SEM images of top random Ag nanoparticles (c) before and (d) after an extra 5-nm-thick NPs deposition. The scale bar is $200 \mathrm{~nm}$. Red dotted squares: areas loaded for simulation. White dotted square: zoom-in SEM images of the surface morphology before and after the second-step NP deposition. (e) Modeled electric field enhancement distribution among NPs in the red dotted squares in (c) and (d) at normal incidence at $\lambda=1000 \mathrm{~nm}$, polarized in the $\mathrm{x}$ direction. (f) Measured SHG intensities and SHG output/ input ratios (blue dashed curve) versus excitation wavelengths obtained with the broadband metasurface after a multi-step deposition process.

red dotted square) to model the spatial distribution of the electric field at normal incidence at $\lambda=1000 \mathrm{~nm}$, with x-polarization. As shown in Fig. 4(e), more hot spots are obtained between large and small NPs, and with stronger localized field intensities. The incident light is mainly localized at edges of $\mathrm{Ag}$ islands, which is the major mechanism for the proposed SHG enhancement (see Section 4 in the supporting material for detailed analysis of electric field distribution). We then characterized the SHG of the same area on the sample. As shown in Fig. 4(f), the broadband super absorbing metasurface after a second-step deposition process exhibits much stronger SHG intensities, indicating a further enhanced light-matter interaction by introducing smaller gaps over a larger area.

Enhanced SHG with $\mathrm{TiO}_{2}$ films. Finally, by integrating an optically transparent material with strong optical nonlinearity into the super absorbing metasurface with the fine particle deposition, SHG signals are expected to be further enhanced due to the combined effects of both the nonlinear material and localized field enhancement from optical nanoantennas on the metasurface. Titanium dioxide $\left(\mathrm{TiO}_{2}\right)$ was chosen for this purpose since it has a high nonlinear refractive index that is 30 times that of silica ${ }^{46}$, while still being transparent in the visible and NIR spectral regions due to its large band gap of $3.1 \mathrm{eV}^{47}$. We employed atomic layer deposition to deposit a $14 \mathrm{~nm} \mathrm{TiO}{ }_{2}$ film at $150^{\circ} \mathrm{C}$ to cover the large and small NPs on the metasurface (see Methods for details of fabrication), as illustrated in Fig. 5(a). High temperatures can facilitate the crystallization of $\mathrm{TiO}_{2}$ into the polycrystalline-anatase phase ${ }^{48}$. Therefore, the super absorbing metasurface with the $\mathrm{TiO}_{2}$ film was then thermally annealed at $400^{\circ} \mathrm{C}$ for 1 hour. As shown by the green curve in Fig. 5(b), a broader and higher absorption was obtained with $>80 \%$ absorption from $435 \mathrm{~nm}$ to $1100 \mathrm{~nm}$ (or $>90 \%$ absorption from $466 \mathrm{~nm}$ to $1100 \mathrm{~nm}$ ). This enhancement in absorption should be attributed to the resonance of the three-layered rMDM super absorbing metasurface, which is sensitive to the dielectric environment ${ }^{49}$ : when we coated the top metallic NPs with a thin film $\mathrm{TiO}_{2}$ layer, its dielectric environment was changed. Therefore, although the $\mathrm{TiO}_{2}$ does not absorb visible and IR light, the resonant condition was changed. The light trapping and optical absorption still occurred within the metallic NP layer.

It is well-known that the symmetry of materials is inherently broken at the surface. Due to the anisotropy of the electric field at the interface, the potential landscape experienced by an atom at the interface is non-symmetric, leading to a surface mediated second-order susceptibility ${ }^{5,50}$. Thus, for a thin film $\mathrm{TiO}_{2}$ over metal islands (a system with a high interfacial area), significant $\mathrm{SHG}$ occurs at these metal- $\mathrm{TiO}_{2}$ interfaces. Although the morphology of metasurface changed during the final thermal annealing process for $\mathrm{TiO}_{2}$ (see discussion in Section 5 in the supporting material), this additional thin $\mathrm{TiO}_{2}$ layer facilitates much stronger SHG intensities, indicating a further enhanced light-matter interaction by introducing nonlinear materials, as shown in Fig. 5(c). For clarity, 
(a)

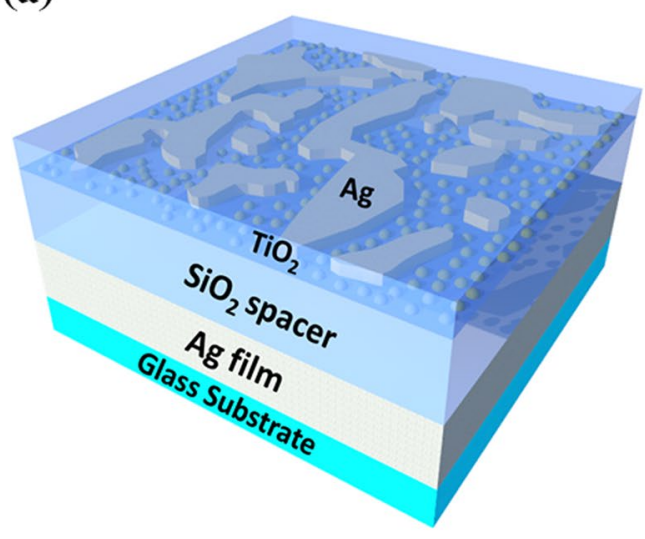

(c)

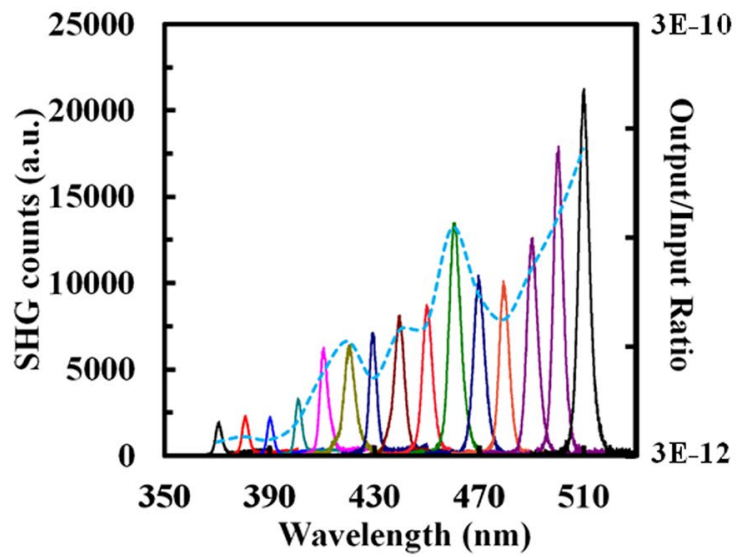

(b) 1

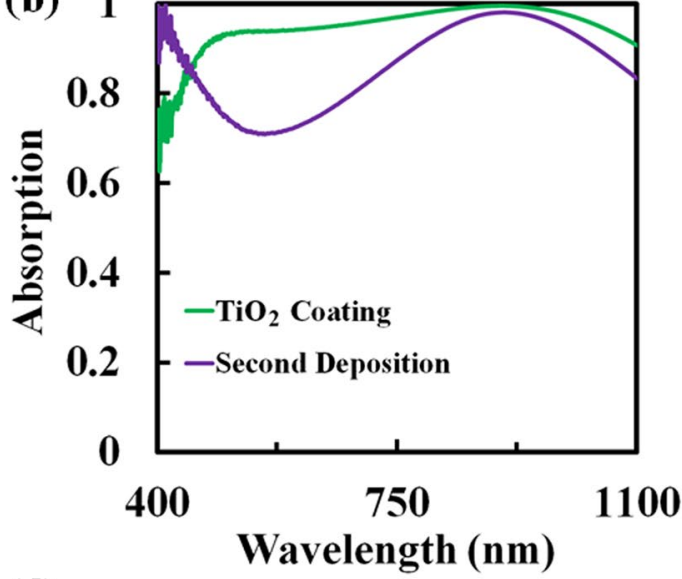

(d)

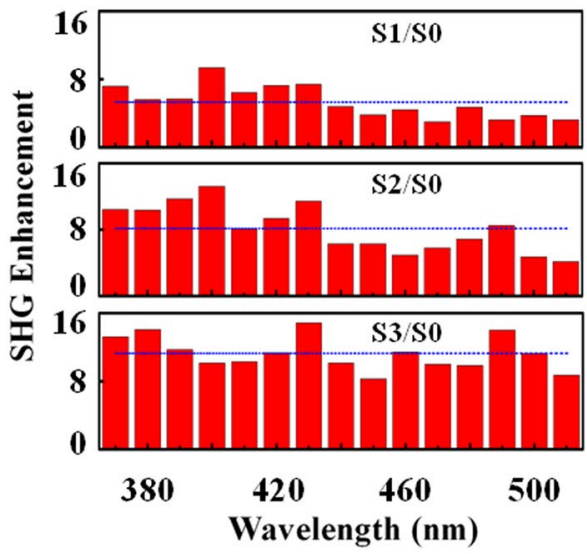

Figure 5. (a) Schematic of the designed three-layered absorbing metasurface after the multi-step deposition and $\mathrm{TiO}_{2}$ film coating. (b) Absorption spectra of the three-layered absorber with a second-step deposition process before (purple curve) and after $\mathrm{TiO}_{2}$ film coating (green curve). (c) Measured SHG intensities and SHG output/input ratios (blue dashed curve) versus incident wavelengths obtained with the broadband metasurface after the multi-step deposition and $\mathrm{TiO}_{2}$ film coating. (d) Spectroscopic enhancement of SHG from the broadband metasurface S1, the metasurface after the fine-particle deposition S2, and the metasurface after fine-particle deposition and $\mathrm{TiO}_{2}$ film coating $\mathrm{S} 3$ compared with that from the reference sample S0. Blue dotted lines: The averaged SHG enhancement over the entire wavelength region.

Fig. 5(d) shows a comparison of spectroscopic enhancement of SHG from different samples compared with the reference sample (i.e., a single layer of deposited NPs on the glass substrate). The reference sample, the broadband metasurface, the metasurface after the fine-particle deposition, and the metasurface after both fine particle deposition and $\mathrm{TiO}_{2}$ film coating are denoted as $\mathrm{S} 0, \mathrm{~S} 1, \mathrm{~S} 2$, and S3, respectively. This comparison clearly demonstrates the improved nonlinear optical properties of the broadband metasurface structure by introducing smaller gaps and $\mathrm{TiO}_{2}$ films. Furthermore, the measured SHG output/input ratio (defined as the measured $\mathrm{P}(2 \omega) / \mathrm{P}(\omega)$ ) of sample S3 at an excitation wavelength of $1020 \mathrm{~nm}$ is $2.14 \times 10^{-10}$ with a pumping intensity of only $6 \mathrm{~kW} / \mathrm{cm}^{2}$. In comparison, previously demonstrated nonlinear optical metasurfaces at infrared/visible/ultraviolet wavelengths required a much higher peak power intensity to produce an SHG conversion efficiency of only $10^{-117,21,51}$. Even though some work reported higher SHG efficiency of up to $10^{-9}$, the top-down lithographic techniques used in these works still impose serious cost barriers for practical applications ${ }^{24,52,53}$.

\section{Conclusion}

In conclusion, we reported a scalable, low cost and broadband super absorbing metasurface substrate for strong field localization and enhanced surface enhance nonlinear optical processes. By manipulating the morphology and composite of the top random nanoantenna layer, an ultra-brandband light trapping was experimentally demonstrated in the wavelength range from $435 \mathrm{~nm}$ to $1100 \mathrm{~nm}$ with strongly localized field. This new plasmonic nonlinear light generation structure has achieved high SHG enhancement by introducing a second-step metal particle deposition process and a nonlinear $\mathrm{TiO}_{2}$ film, compared with the reference sample of only Ag NPs on a bare glass substrate. Importantly, this broadband light trapping metasurface structure is completely lithography free, suitable for future large area roll-to-roll deposition processes for inexpensive nanomanufacturing. In addition to providing new understanding of broadband light trapping and field localization, this work may 
open avenues toward new applications in energy harvesting ${ }^{20,54-56}$, conversion ${ }^{57-60}$ and surface enhanced Raman spectroscopy ${ }^{61-63}$.

\section{Methods}

Metasurface Fabrication. $\quad 2.5 \times 7.5 \mathrm{~cm}^{2}$ microscope glass slides were sequentially sonicated in acetone, isopropyl alcohol (IPA), and deionized water for 15 minutes. The fabrication of metal/dielectric/metal metasurface began with a 200-nm-thick Ag ground plate deposited on the glass slide using AJA sputtering/e-beam evaporation dual chamber hybrid thin film deposition system. During the deposition, the Argon pressure in the chamber was set to 3 mTorr with no heating on the substrate. The Ag deposition rate was controlled by the sputter voltage and direct current power at $2.6 \AA / \mathrm{sec}$. Then the $70 \mathrm{~nm} \mathrm{SiO}_{2}$ film was deposited using an electron-beam evaporation system (BOC Edwards Auto 500 system) with the deposition rate of $1 \AA$ Asec under vacuum $\left(7.0 \times 10^{-6}\right.$ Torr $)$. For the top layer, $14 \mathrm{~nm}$-thick Ag film was deposited with the deposition rate of $2.6 \AA / \mathrm{sec}$ in the chamber of AJA sputtering system. Next, the Ag mesh network was transformed to isolated nanoparticles by introducing a thermal annealing process at the temperature of $200^{\circ} \mathrm{C}$ (i.e., Fig. 1(b)) for 60 minutes. In the second-step direct deposition process, a $5 \mathrm{~nm}$-thick $\mathrm{Ag}$ film was deposited with a deposition rate of $2.6 \AA / \mathrm{sec}$ in the chamber of AJA sputtering system. Finally, a $14 \mathrm{~nm}$-thick $\mathrm{TiO}_{2}$ film was deposited using Ultratech/CambridgeNanotech atomic layer deposition system at $150^{\circ} \mathrm{C}$.

Characterization. SEM images were taken using Zeiss CrossBeam ${ }^{\circledR}$ Workstation system. The reflection/ absorption spectra of metasurfaces were characterized using a microscopic Fourier transform infrared spectroscopy (Bruker, VETEX $70+$ Hyperion 1000) and UV/Vis/NIR spectrophotometer (Perkin-Elmer Lambda 750). The observation area for each sample was set to $50 \mu \mathrm{m} \times 50 \mu \mathrm{m}$.

SHG experimental setup. Laser pulses (red beam in Fig. 2(a)) were generated by using a broad-band Ti:sapphire oscillator (Spectra-Physics MaiTai) and an optical parametric oscillator (Spectra-Physics Inspire 100) with $80 \mathrm{fs}$ duration and $80 \mathrm{MHz}$ repetition rate, then passed through a long pass filter (ET542lp Chroma) before being directed into a home-made upright microscope. The laser beam was vertically focused on the metasurface by a CaF2 UV objective (LMU-15X-UVB NA0.32 Thorlabs). The fundamental wavelength was scanned from $740 \mathrm{~nm}$ to $1020 \mathrm{~nm}$ with $20 \mathrm{~nm}$ increments. The SHG signal (blue beam in Fig. 2(a)) was collected in the backward direction, extracted by a long pass dichroic mirror (DMLP567 Thorlabs, 340dclp Chroma) and filters (D350-50x/ ET385-70X/ET700sp Chroma). A spectrograph (Acton SpectraPro, SP-2500) equipped with a liquid nitrogen cooled CCD is used to analyze the SHG spectra. The pump power of the fundamental beam at all wavelengths was set to $1.2 \mathrm{~mW}$ as measured at the sample position. SHG spectra were measured by scanning the fundamental wavelengths.

\section{References}

1. Lapine, M., Shadrivov, I. V. \& Kivshar, Y. S. Colloquium: Nonlinear metamaterials. Reviews of Modern Physics 86, 1093-1123 (2014).

2. Leuthold, J., Koos, C. \& Freude, W. Nonlinear silicon photonics. Nature Photonics 4, 535-544 (2010).

3. Leon, I. D., Shi, Z., Liapis, A. C. \& Boyd, R. W. Measurement of the complex nonlinear optical response of a surface plasmonpolariton. Optics Letters 39, 2274-2277 (2014).

4. Boyd, R. W., Shi, Z. \& Leon, I. D. The third-order nonlinear optical susceptibility of gold. Optics Communications 326, 74-79 (2014).

5. Kauranen, M. \& Zayats, A. V. Nonlinear plasmonics. Nature Photonics 6, 737-748 (2012).

6. Litchinitser, N. M. \& Sun, J. Optical meta-atoms: Going nonlinear. Science 350, 1033-1034 (2015).

7. Kang, L. et al. Electrifying photonic metamaterials for tunable nonlinear optics. Nature Communications 5, 4680 (2014).

8. Park, I.-Y. et al. Plasmonic generation of ultrashort extreme-ultraviolet light pulses. Nature Photonics 5, 677-681 (2011).

9. Kim, S. et al. High-harmonic generation by resonant plasmon field enhancement. Nature 453, 757-760 (2008).

10. Klein, M. W., Enkrich, C., Wegener, M. \& Linden, S. Second-harmonic generation from magnetic metamaterials. Science 313, 502-504 (2006)

11. Liu, S. et al. Polarization-independent multiple Fano resonances in plasmonic nonamers for multimode-matching enhanced multiband second-harmonic generation. ACS Nano 10, 1442-1453 (2016).

12. Ding, W., Zhou, L. \& Chou, S. Y. Enhancement and electric charge-assisted tuning of nonlinear light generation in bipolar plasmonics. Nano Letters 14, 2822-2830 (2014).

13. Linnenbank, H., Grynko, Y., Förstner, J. \& Linden, S. Second harmonic generation spectroscopy on hybrid plasmonic/dielectric nanoantennas. Light: Science \& Applications 5, e16013 (2016).

14. Lee, J. et al. Giant nonlinear response from plasmonic metasurfaces coupled to intersubband transitions. Nature 511, 65 (2014).

15. Zhu, J. Theoretical study of the tunable second-harmonic generation SHG, enhancement factor of gold nanotubes. Nanotechnology 18, 225702 (2007).

16. Liu, K. et al. A large-scale lithography-free metasurface with spectrally tunable super absorption. Nanoscale 6, 5599-5605 (2014).

17. Zhang, N. et al. Ultrabroadband metasurface for efficient light trapping and localization: A universal surface-enhanced Raman spectroscopy substrate for "all" excitation wavelengths. Advanced Materials Interfaces 2, 1500142 (2015).

18. Rodrigues, S. P. \& Cai, W. Nonlinear optics: Tuning harmonics with excitons. Nature Nanotechnology 10, 387-388 (2015).

19. Søndergaard, T. et al. Plasmonic black gold by adiabatic nanofocusing and absorption of light in ultra-sharp convex grooves. Nature Communications 3, 969 (2012).

20. Schuller, J. A. et al. Plasmonics for extreme light concentration and manipulation. Nature Materials 9, 193-204 (2010).

21. Feth, N. et al. Second-harmonic generation from complementary split-ring resonators. Optics Letters 33, 1975-1977 (2008).

22. Lassiter, J. B. et al. Third-harmonic generation enhancement by film-coupled plasmonic stripe resonators. ACS Photonics $\mathbf{1}$, 1212-1217 (2014).

23. Metzger, B. et al. Doubling the efficiency of third harmonic generation by positioning ITO nanocrystals into the hot-spot of plasmonic gap-antennas. Nano Letters 14, 2867-2872 (2014).

24. Walsh, G. F. \& Negro, L. D. Enhanced second harmonic generation from Au nanoparticle arrays by femtosecond laser irradiation. Nanoscale 5, 7795-7799 (2013).

25. Arjmand, A., Abolghasem, P., Han, J. \& Helmy, A. S. Interface modes for monolithic nonlinear photonics. J. Opt. Soc. Am. B 32, 577-587 (2015)

26. Celebrano, M. et al. Mode matching in multiresonant plasmonic nanoantennas for enhanced second harmonic generation. Nature Nanotechnology 10, 412-417 (2015). 
27. Stockman, M. I., Bergman, D. J., Anceau, C., Brasselet, S. \& Zyss, J. Enhanced second-harmonic generation by metal surfaces with nanoscale roughness: Nanoscale dephasing, depolarization, and correlations. Physical Review Letters 92, 057402 (2004).

28. Anceau, C., Brasselet, S., Zyss, J. \& Gadenne, P. Local second-harmonic generation enhancement on gold nanostructures probed by two-photon microscopy. Optics Letters 28, 713-715 (2003).

29. Nappa, J. et al. Electric dipole origin of the second harmonic generation of small metallic particles. Physical Review B 71, 165407 (2005).

30. Salomon, A., Zielinski, M., Kolkowski, R., Zyss, J. \& Prior, Y. Size and shape resonances in second harmonic generation from silver nanocavities. The Journal of Physical Chemistry C 117, 22377-22382 (2013).

31. Harfouch, Y. E. et al. Hyper-Rayleigh scattering from gold nanorods. The Journal of Physical Chemistry C 118, 609-616 (2014).

32. Hyun, J. K. et al. Enhanced second harmonic generation by coupling to exciton ensembles in Ag-coated ZnO nanorods. ACS Photonics 2, 1314-1319 (2015).

33. Raza, S., Bozhevolnyi, S. I., Wubs, M. \& Mortensen, N. A. Nonlocal optical response in metallic nanostructures. Journal of Physics: Condensed Matter 27, 183204 (2015).

34. Wang, T. \& Nijhuis, C. A. Molecular electronic plasmonics. Applied Materials Today 3, 73-86 (2016).

35. Ciraci, C. et al. Probing the ultimate limits of plasmonic enhancement. Science 337, 1072-1074 (2012).

36. Nijs, B. D. et al. Unfolding the contents of sub-nm plasmonic gaps using normalising plasmon resonance spectroscopy. Faraday Discussions 178, 185-193 (2015).

37. Müller, K. et al. Atomic electric fields revealed by a quantum mechanical approach to electron picodiffraction. Nature Communications 5, 5653 (2014).

38. Chikkaraddy, R. et al. Single-molecule strong coupling at room temperature in plasmonic nanocavities. Nature 535, 127-130 (2016).

39. Zhu, W. et al. Quantum mechanical effects in plasmonic structures with subnanometre gaps. Nature Communications 7, 11495 (2016).

40. Roelli, P., Galland, C., Piro, N. \& Kippenberg, T. J. Molecular cavity optomechanics as a theory of plasmon-enhanced Raman scattering. Nature Nanotechnology 11, 164-169 (2015).

41. Zhu, W. \& Crozier, K. B. Quantum mechanical limit to plasmonic enhancement as observed by surface-enhanced Raman scattering. Nature Communications 5, 5228 (2014).

42. Benz, F. et al. Single-molecule optomechanics in "picocavities". Science 354, 726-729 (2016).

43. Serrano, A., Rodríguez de la Fuente, O. \& García, M. A. Extended and localized surface plasmons in annealed Au films on glass substrates. Journal of Applied Physics 108, 074303 (2010).

44. Hafezian, S., Baloukas, B. \& Martinu, L. Percolation threshold determination of sputtered silver films using Stokes parameters and in situ conductance measurements. Applied Optics 53, 5367-5374 (2014).

45. Liu, W., Oulton, R. F. \& Kivshar, Y. S. Geometric interpretations for resonances of plasmonic nanoparticles. Scientific Reports 5, 12148 (2015)

46. Evans, C. C., Bradley, J. D. B., Martì-Panameño, E. A. \& Mazur, E. Mixed two- and three-photon absorption in bulk rutile $\mathrm{TiO}_{2}$, around $800 \mathrm{~nm}$. Optics Express 20, 3118-3128 (2012).

47. Wendt, S. et al. The Role of Interstitial Sites in the Ti3d Defect State in the Band Gap of Titania. Science 320, 1755-1759 (2008).

48. Jin, C., Liu, B., Lei, Z. \& Sun, J. Structure and photoluminescence of the $\mathrm{TiO}_{2}$ films grown by atomic layer deposition using tetrakisdimethylamino titanium and ozone. Nanoscale Research Letters 10, 95 (2015).

49. Zhang, N. et al. Reversibly tunable coupled and decoupled super absorbing structures. Applied Physics Letters 108, 091105 (2016).

50. Smirnova, D. \& Kivshar, Y. S. Multipolar nonlinear nanophotonics. Optica 3, 1241-1255 (2016).

51. Belardini, A. et al. Tailored second harmonic generation from self-organized metal nano-wires arrays. Optics Express 17, 3603-3609 (2009).

52. Zhang, Y., Grady, N. K., Ayala-Orozco, C. \& Halas, N. J. Three-dimensional nanostructures as highly efficient generators of second harmonic light. Nano Letters 11, 5519-5523 (2011).

53. Aouani, H. et al. Multiresonant broadband optical antennas as efficient tunable nanosources of second harmonic light. Nano Letters 12, 4997-5002 (2012)

54. Boriskina, S. V., Ghasemi, H. \& Chen, G. Plasmonic materials for energy: From physics to applications. Materials Today 16, 375-386 (2013).

55. Kawawaki, T., Asakura, A. \& Tatsuma, T. Branched Au nanoparticles as light-harvesting antennae for photosensitized reactions. ChemNanoMat 2, 74-78 (2016).

56. Callahan, D. M., Munday, J. N. \& Atwater, H. A. Solar cell light trapping beyond the ray optic limit. Nano Letters 12, 214-218 (2012).

57. Gan, Q., Bartoli, F. J. \& Kafafi, Z. H. Plasmonic-enhanced organic photovoltaics: Breaking the $10 \%$ efficiency barrier. Advanced Materials 25, 2385-2396 (2013).

58. Atwater, H. A. \& Polman, A. Plasmonics for improved photovoltaic devices. Nature Materials 9, 205-213 (2010).

59. Kim, G. M. \& Tatsuma, T. Semitransparent solar cells with ultrasmooth and low-scattering Perovskite thin films. J. Phys. Chem. C 120, 28933-28938 (2016).

60. Gong, T., Krayer, L. \& Munday, J. N. Design concepts for hot carrier-based detectors and energy converters in the near ultraviolet and infrared. J. Photon. Energy 6, 042510 (2016).

61. Stiles, P., Dieringer, J., Shah, N. C. \& Van Duyne, R. P. Surface-enhanced Raman spectroscopy. Annual Review of Analytical Chemistry 1, 601-626 (2008).

62. Sharma, B., Frontiera, R. R., Henry, A.-I., Ringe, E. \& Van Duyne, R. P. SERS: materials, applications, and the future. Materials Today 15, 16-25 (2012)

63. Eftekhari, F., Lee, A., Kumacheva, E. \& Helmy, A. S. Examining metal nanoparticle surface chemistry using hollow-core, photoniccrystal, fiber-assisted SERS. Optics Letters 37, 680-682 (2012).

\section{Acknowledgements}

Q. Gan acknowledges funding support from National Science Foundation (grant no. ECCS1507312, CBET1445934 and ECCS1425648). N. Zhang acknowledges the financial support from Chinese Scholarship Council (CSC). K. Shi acknowledges the funding support from the National Science Foundation of China (NSFC\#61322509) and the Ministry of Science and Technology of China (National Basic Research Program of China under Grant No. 2013CB921904, State Key Instrument Development Program \#2012YQ140005).

\section{Author Contributions}

N.Z., Z.J. and A.R.C. contributed equally to this work. Q.G., A.N.C. and K.S. conceived the idea and supervised the project. N.Z., A.R.C., H.S. and D.J. designed, fabricated, and characterized the patterned super absorbing metasurfaces. Z.J., A.R.C. and T.Z. performed the second harmonic generation experiments and data analysis. N.Z., X.Z. and B.C. performed the numerical modeling and data analysis. N.Z., Z.J., A.R.C., Q.G., A.N.C. and K.S. wrote the manuscript. All reviewed the manuscript. 


\section{Additional Information}

Supplementary information accompanies this paper at doi:10.1038/s41598-017-04688-4

Competing Interests: The authors declare that they have no competing interests.

Publisher's note: Springer Nature remains neutral with regard to jurisdictional claims in published maps and institutional affiliations.

(c) (i) Open Access This article is licensed under a Creative Commons Attribution 4.0 International License, which permits use, sharing, adaptation, distribution and reproduction in any medium or format, as long as you give appropriate credit to the original author(s) and the source, provide a link to the Creative Commons license, and indicate if changes were made. The images or other third party material in this article are included in the article's Creative Commons license, unless indicated otherwise in a credit line to the material. If material is not included in the article's Creative Commons license and your intended use is not permitted by statutory regulation or exceeds the permitted use, you will need to obtain permission directly from the copyright holder. To view a copy of this license, visit http://creativecommons.org/licenses/by/4.0/.

(C) The Author(s) 2017 